\title{
Ram pressure stripping in the Virgo Cluster
}

\author{
C. Verdugo ${ }^{1}$, F. Combes ${ }^{1,2}$, K. Dasyra ${ }^{1,3}$, P. Salomé ${ }^{1}$, and J. Braine ${ }^{4}$ \\ 1 Observatoire de Paris, LERMA, CNRS UMR 8112, 61 Av. de l'Observatoire, 75014 Paris, France \\ e-mail: celia.verdugo@obspm.fr \\ 2 Collège de France, 11 place Marcelin Berthelot, 75005 Paris, France \\ 3 Department of Astrophysics, Astronomy \& Mechanics, Faculty of Physics, University of Athens, \\ 15784 Panepistimiopolis Zografos, Greece \\ ${ }^{4}$ Univ. Bordeaux, Laboratoire d'Astrophysique de Bordeaux, CNRS UMR 5804, 33270 Floirac, France
}

Received 18 May 2015 / Accepted 9 July 2015

\begin{abstract}
Gas can be violently stripped from their galaxy disks in rich clusters, and be dispersed over $100 \mathrm{kpc}$-scale tails or plumes. Young stars have been observed in these tails, suggesting they are formed in situ. This will contribute to the intracluster light, in addition to tidal stripping of old stars. We want to quantify the efficiency of intracluster star formation. We present $\mathrm{CO}(1-0)$ and $\mathrm{CO}(2-1)$ observations, made with the IRAM-30 m telescope, towards the ram-pressure stripped tail northeast of NGC 4388 in Virgo. We selected HII regions found all along the tails, together with dust patches, as observing targets. We detect molecular gas in 4 positions along the tail, with masses between $7 \times 10^{5}$ to $2 \times 10^{6} M_{\odot}$. Given the large distance from the NGC 4388 galaxy, the molecular clouds must have formed in situ, from the HI gas plume. We compute the relation between surface densities of star formation and molecular gas in these regions, and find that the star formation has very low efficiency. The corresponding depletion time of the molecular gas can be up to $500 \mathrm{Gyr}$ and more. Since this value exceeds a by far Hubble time, this gas will not be converted into stars, and will stay in a gaseous phase to join the intracluster medium.
\end{abstract}

Key words. galaxies: evolution - galaxies: clusters: individual: Virgo - galaxies: clusters: intracluster medium - galaxies: ISM galaxies: interactions

\section{Introduction}

In overdense cluster environments, galaxies are significantly transformed through different tidal interactions, like those caused by other galaxies, the cluster as a whole (e.g. Merritt 1984; Tonnesen et al. 2007), and those with the intracluster medium (ICM), which strips them from their gas content. This ram-pressure stripping (RPS) process has been described by Gunn \& Gott (1972) and simulated by many groups (Quilis et al. 2000; Vollmer et al. 2001; Roediger \& Hensler 2005; Jáchym et al. 2007). Evidence of stripping has been observed in many cases (Kenney et al. 2004; Chung et al. 2007; Sun et al. 2007; Vollmer et al. 2008). The RPS and tidal interactions can disperse the interstellar medium (ISM) of galaxies at large distance, up to $100 \mathrm{kpc}$ scales, as shown by the spectacular tail of ionized gas in Virgo (Kenney et al. 2008).

What is the fate of the stripped gas? According to the timescale of the ejection, the relative velocity of the ICM-ISM interaction, and the environment, this gas could be first seen as neutral atomic gas (Chung et al. 2009; Scott et al. 2012; Serra et al. 2013), then ionized gas detected in $\mathrm{H} \alpha$ (Gavazzi et al. 2001; Cortese et al. 2007; Yagi et al. 2007; Zhang et al. 2013), and finally is heated to X-ray gas temperatures (e.g. Machacek et al. 2005, Sun et al. 2010). In rarer cases, stripped gas can be seen as dense and cold molecular gas, detected as carbon monoxide (CO) emission (Vollmer et al. 2005; Dasyra et al. 2012; Jáchym et al. 2014). The presence of these dense molecular clumps might appear surprising, since the RPS should not be able to drag them out of their galaxy disks (Nulsen 1982; Kenney \& Young 1989). However, they could reform quickly in the tail. Unless they are self-shielded (e.g., Machacek et al. 2004; Fabian et al. 2006; Tamura et al. 2009), the survival of these clouds in the hostile ICM environment, with temperature $10^{7} \mathrm{~K}$ and destructive X-rays (e.g., Machacek et al. 2004; Fabian et al. 2006; Tamura et al. 2009) is puzzling. The presence of cold molecular gas is also observed in rich galaxy clusters with cool cores. Here also a multiphase gas has been detected, in $\mathrm{CO}, \mathrm{H} \alpha$, $\mathrm{X}$-rays, and also the strongest atomic cooling lines (Edge et al. 2010). Ionized gas, together with warm atomic and molecular gas and cold molecular gas clouds, coexist in spatially resolved filaments around the brightest cluster galaxy, such as in the spectacular prototype Perseus A (Conselice et al. 2001; Salomé et al. 2006, 2011; Lim et al. 2012).

The survival of molecular clouds was also observed by Braine et al. (2000) in several tidal tails, and in particular in the interacting system Arp 105 (dubbed the Guitar), embedded in the X-ray emitting medium of the Abell 1185 Cluster (Mahdavi et al. 1996). Again, the formation in situ of the molecular clouds is favored (Braine et al. 2000). In the Stephan's Quintet compact group, where X-ray gas and star formation have been observed in between galaxies (O'Sullivan et al. 2009), the shock has been so violent $\left(1000 \mathrm{~km} \mathrm{~s}^{-1}\right)$ that $\mathrm{H}_{2}$ molecules are formed and provide the best cooling agent through mid-infrared radiation (Cluver et al. 2010). In this shock, multiphases of gas coexist, from cold dense molecular gas to X-ray gas.

Does this gas form stars? In usual conditions, inside galaxy disks, the star formation is observed to depend on the amount of molecular gas present (e.g., Bigiel et al. 2008; Leroy et al. 2013). A Schmidt-Kennicutt (S-K) relation is observed, roughly linear, between the surface densities of star formation and molecular 
gas, leading to a depletion timescale $\left(\tau_{\text {dep }}=\Sigma_{\text {gas }} / \Sigma_{\mathrm{SFR}}\right)$ of 2 Gyr. This relation, however, does not apply to specific regions or circumstances, such as galaxy centers (Casasola et al. 2015), outer parts of galaxies and extended UV disks (Dessauges-Zavadsky et al. 2014), or low surface brightness galaxies (Boissier et al. 2008). Little is known about star formation in gas clouds stripped from galaxies in rich clusters. Boissier et al. (2012) have put constraints on this process, concluding in a very low star formation efficiency, lower by an order of magnitude than observed in normal galaxy disks, and even lower than outer parts of galaxies or in low surface brightness galaxies. It is useful to better constrain this efficiency, given the large amount of intracluster light (ICL) observed today (e.g., Feldmeier et al. 2002; Mihos et al. 2005). These stars could come from tidal stripping of old stars formed in galaxy disks, or a large fraction of these stars could have formed in situ from ram-pressure stripped gas. More intracluster star formation could have formed in the past (DeMaio et al. 2015). The origin of the ICL could bring insight on the relative role of galaxy interactions during the cluster formation, or cluster processing after relaxation.

One of the most suited environments to probe the survival of molecular gas and the efficiency of star formation under extreme ram-pressure conditions is the RPS tail north of NGC 4388 in the Virgo Cluster south of M86, where X-ray gas has been mapped (Iwasawa et al. 2003) and young stars have been found (Yagi et al. 2013). It is located at about $400 \mathrm{kpc}$ in projection from the cluster center M87. NGC 4388 is moving at a relative velocity redshifted by $1500 \mathrm{~km} \mathrm{~s}^{-1}$ with respect to M87, and more than $2800 \mathrm{~km} \mathrm{~s}^{-1}$ with respect to the M86 group. This strong velocity may explain the violent RPS, the high HI deficiency of NGC 4388 (Cayatte et al. 1990), and the large ( 35 kpc) emission-line region found by Yoshida et al. (2002), northeast of the galaxy. The ionized gas has a mass of $10^{5} M_{\odot}$, and is partly excited by the ionizing radiation of the Seyfert 2 nucleus in NGC 4388. The RPS plume is even more extended in HI (Oosterloo \& van Gorkom 2005), up to $110 \mathrm{kpc}$, with a mass of $3.4 \times 10^{8} M_{\odot}$. Gu et al. (2013) found neutral gas in absorption in X-ray, with column densities $2-3 \times 10^{20} \mathrm{~cm}^{-2}$, revealing that the RPS tail is in front of M86. The large ratio between hot and cold gas in the clouds means that significant evaporation has occurred. Yagi et al. (2013) find star-forming regions in the plume at 35 and $66 \mathrm{kpc}$ from NGC 4388, with solar metallicity and age 6 Myr. Since these stars are younger than the RPS event, they must have formed in situ.

In the present paper, we present $\mathrm{CO}$ detections in the rampressure stripped gas northeast of NGC 4388. In a previous paper, we already found molecular gas in a ionized gas tail south of M86 (Dasyra et al. 2012), and discussed its survival conditions. Here we study the link between new stars formed and molecular gas to derive the star formation efficiency. In the RPS plume, a significant fraction of the $\mathrm{H} \alpha$ emission could originate from the ionized gas in the outer layers of molecular clouds (Ferland et al. 2009). This makes the H $\alpha$ lumps good tracers of star formation in an RPS tail for the purposes of probing the efficiency of the process of formation of intracluster stars. Section 2 presents the IRAM-30 m observations, Sect. 3 the results obtained, which are discussed in Sect. 4.

In the following, we assume a distance of $17.5 \mathrm{Mpc}$ to the Virgo Cluster (Mei et al. 2007).

\section{Observations and data reduction}

We completed CO observations along the HI plume (Oosterloo \& van Gorkom 2005) connecting NGC 4388 and M86 with
Table 1. Targets and observations.

\begin{tabular}{ccccc}
\hline \hline Source & $\begin{array}{c}\text { RA(J2000) } \\
{\left[{ }^{\mathrm{h} . \mathrm{m} . \mathrm{s}]}\right.}\end{array}$ & $\begin{array}{c}\text { Dec(J2000) } \\
{\left[{ }^{\mathrm{deg} . \mathrm{m}: \mathrm{s}}\right]}\end{array}$ & $\begin{array}{c}\text { Hel. vel. } \\
{\left[\mathrm{km} \mathrm{s}^{-1}\right]}\end{array}$ & $\begin{array}{c}\text { ON + OFF } \\
{[\mathrm{h}]}\end{array}$ \\
\hline Source-1 & $12: 26: 01.3$ & $12: 42: 30.1$ & +2500 & 4.2 \\
Source-2 & $12: 26: 04.9$ & $12: 45: 16.7$ & +2500 & 2.9 \\
Source-3 & $12: 26: 25.4$ & $12: 50: 53.6$ & +2200 & 3.1 \\
Source-5 & $12: 26: 24.5$ & $12: 58: 14.6$ & +2000 & 3.9 \\
HaR-2 & $12: 26: 13.7$ & $12: 51: 36.9$ & +2230 & 7.2 \\
HaR-3-4 & $12: 26: 13.5$ & $12: 43: 03.7$ & +2500 & 3.8 \\
\hline HaR-2-1 & $12: 26: 16.9$ & $12: 53: 55.6$ & +2230 & 4.5 \\
HaR-2-2 & $12: 26: 16.9$ & $12: 53: 19.3$ & +2230 & 5.6 \\
HaR-2-3 & $12: 26: 16.9$ & $12: 52: 43.1$ & +2230 & 6.2 \\
HaR-2-4 & $12: 26: 15.5$ & $12: 52: 01.1$ & +2230 & 8.0 \\
HaR-1 & $12: 26: 14.6$ & $12: 51: 46.4$ & +2230 & 3.8 \\
NGC 4388 & $12: 25: 46.6$ & $12: 39: 44.0$ & +2550 & 1.2 \\
\hline
\end{tabular}

Notes. Sources in the first 6 rows correspond to run 195-13, and last 6 rows to run 075-14 (although source HaR-2 was observed in both runs). NGC 4388 was observed for calibration purposes. Heliocentric velocities are referencial, taken from Oosterloo \& van Gorkom (2005) along the HI plume.

the IRAM 30-mt telescope at Pico Veleta, Spain, in two separate runs. The first run was part of the project 195-13, with $28 \mathrm{~h}$ of observation, and took place between the 5 th and 8 th of December 2013 with excellent weather conditions $(\tau<0.1$ and a pwv between 0.1 and $3 \mathrm{~mm}$ ). The second run was project 075 14 , with 47 h of observations between June 25th-30th 2014, and had poor to average weather conditions ( $\tau$ between 0.2 and 0.6 and a pwv between 3 and $10 \mathrm{~mm}$ ).

We did all observations with the EMIR receiver in the E0/E2 configuration, allowing us to observe simultaneously $\mathrm{CO}(1-0)$ and $\mathrm{CO}(2-1)$ at 115.271 and $230.538 \mathrm{GHz}$, respectively. The telescope half-power beam widths at these frequencies are $22^{\prime \prime}$ and $11^{\prime \prime}$, respectively. The observing strategy consisted in single $\mathrm{ON}+\mathrm{OFF}$ pointings per each target, with wobbler switching.

We selected targets along the HI plume for having a match of $\mathrm{HI}$ (using the $\mathrm{N}_{\mathrm{HI}}$ map from Oosterloo \& van Gorkom 2005), $\mathrm{H} \alpha$ (with data from Kenney et al. 2008 and Yagi et al. 2013), and $250 \mu$ emission (Herschel SPIRE data from Davies et al. 2012). See Fig. 1. With this criteria six targets where selected for the first run 195-13, and are listed in Table 1 (first six rows). As a result of this run, only two sources showed CO detection: Source-1 and HaR-2. Since HaR-2 is of particular interest because it is so far away from both galaxies and because it has a strong $\mathrm{H} \alpha$ detection (Yagi et al. 2013), this source was chosen as a central target, and we selected another five targets nearby this source for the second run 075-14 (second half of Table 1), following the path of an HI peak (Fig. 1 top right box)

Concerning the spectral resolution of our data, during the observations both FTS and WILMA backends were used simultaneously. The FTS backend has a spectral resolution of $195 \mathrm{kHz}$ and a bandwidth of $32 \mathrm{GHz}$ including both polarizations. At $115 \mathrm{GHz}$ these values correspond to 0.5 and $83200 \mathrm{~km} \mathrm{~s}^{-1}$, and at $230 \mathrm{GHz}$ to 0.25 and $41600 \mathrm{~km} \mathrm{~s}^{-1}$. As for the WILMA backend, we obtained a spectral resolution of $2 \mathrm{MHz}$ and a bandwidth of $16 \mathrm{GHz}$. At $115 \mathrm{GHz}$ this translates to 5.2 and $41600 \mathrm{~km} \mathrm{~s}^{-1}$, and to 2.6 and $20800 \mathrm{~km} \mathrm{~s}^{-1}$ at $230 \mathrm{GHz}$.

We reduced the data using the CLASS software from the GILDAS package. First, a careful inspection of all scans was done to remove bad scans. The approved scans of the same source, $\mathrm{CO}$ line, and backend were averaged with a normal time 


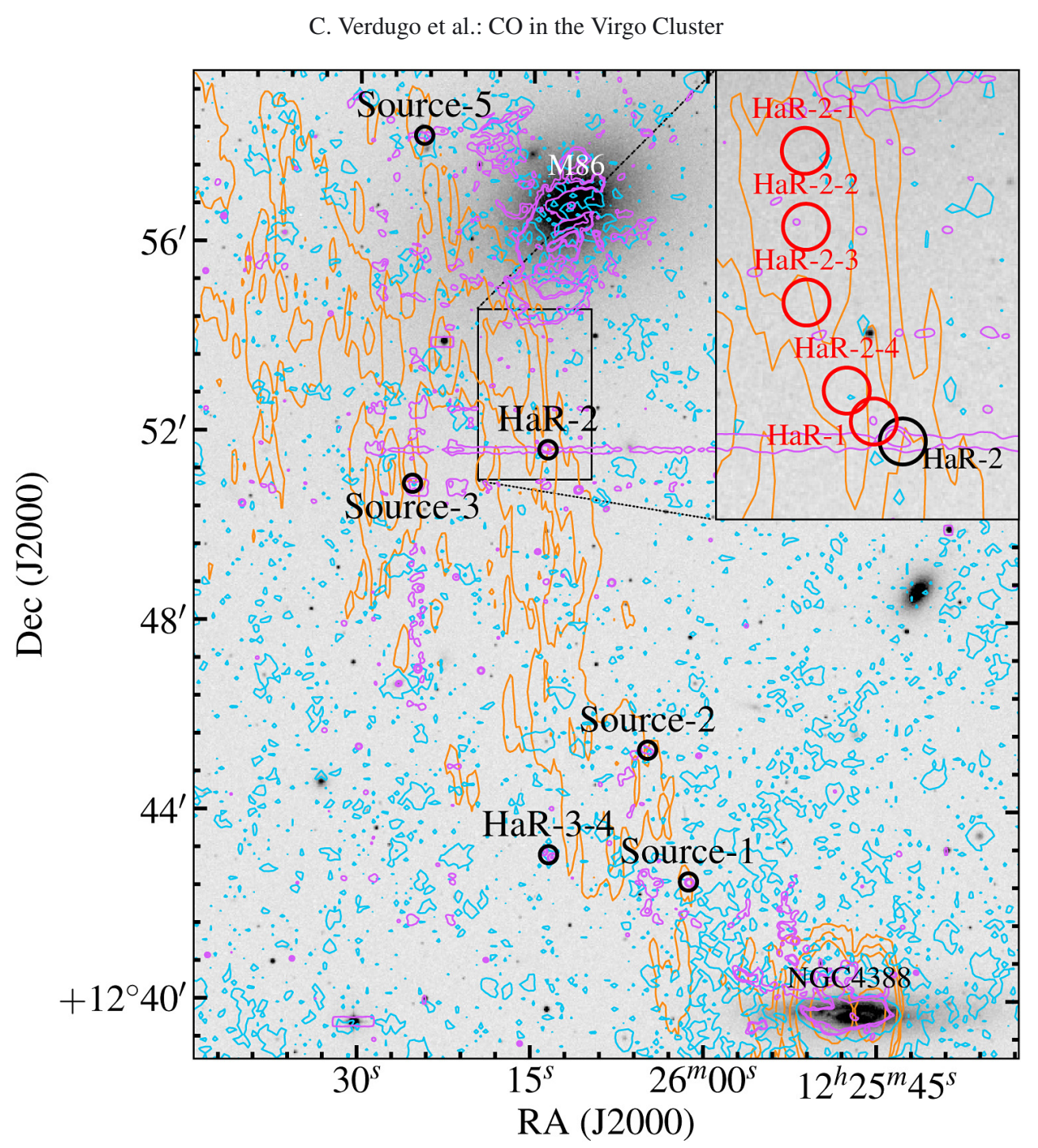

Fig. 1. Targets observed in the Virgo Cluster. Orange: HI contours levels at 1, 5, 10 and $50 \times 10^{19} \mathrm{~cm}^{-2}$ from Oosterloo \& van Gorkom (2005), showing the plume of atomic gas stripped from NGC 4388. Purple: $\mathrm{H} \alpha$ contours levels at 5, 11, and $50 \mathrm{e}^{-} / \mathrm{s}$ (Kenney et al. 2008). Cyan: $250 \mu \mathrm{m}$ contour levels at 0.01 and $0.1 \mathrm{Jy} /$ beam (Davies et al. 2012). In zoomed regions: targets selected close to HaR-2 for run 075-14 (red circles). Circles enclosing targets are $22^{\prime \prime}$ width, as the $\mathrm{CO}(1-0) \mathrm{HPBW}$.

weighting to obtain one spectrum. Then, each spectrum was inspected individually, and in both of its polarizations, to identify a possible $\mathrm{CO}$ emission line. If we found a detection in the spectra of both backends, we chose the best spectrum (in terms of spectral resolution and $\mathrm{S} / \mathrm{N}$ ) as the final spectrum. The selected spectra with $\mathrm{CO}$ emission are presented in Figs. 2 and 3, which contain both polarizations, horizontal and vertical, combined.

Baselines were subtracted with polynomials of order 0 and 1 , depending on the source, and antenna temperatures were corrected by the telescope beam and forward efficiencies ${ }^{1}$ to obtain main beam temperatures. We smoothed the spectra with the hanning method to degrade the velocity resolution until we obtained a value no greater than $1 / 3$ of the FWHM line.

Finally, a simple Gaussian line was fitted to the line candidate. The CLASS fit return the velocity position of the line, its FWHM, the peak temperature and the integrated line intensity.

These spectra and fitting results for sources with $\mathrm{CO}$ detection are presented in Figs. 2 (from the first run) and 3 (from second run) and then in Table 2. For the rest of the sources, with no

\footnotetext{
1 http://wWW.iram.es/IRAMES/mainWiki/

Iram30mEfficiencies
}

visible $\mathrm{CO}$ detections, $3 \sigma$ upper limits for $I_{\mathrm{CO}}$ where calculated from their rms values, assuming a $\Delta v=30 \mathrm{~km} \mathrm{~s}^{-1}$. These limits are presented in Table 3 .

\section{Results}

After selecting the final spectra for every source with a visible detection and fitting their Gaussian profiles, line parameters are calculated and presented in Table 2 . These $\mathrm{CO}$ detections present velocity centroids in the range of $\sim 2200-2500 \mathrm{~km} \mathrm{~s}^{-1}$, and are very consistent with the HI velocities from Table 1 , taken from Oosterloo \& van Gorkom 2005 (their Fig. 2). $\mathrm{H}_{2}$ masses were derived from the $\mathrm{CO}(1-0)$ line intensity, using a Galactic $\mathrm{CO}$ conversion factor of $2.0 \times 10^{20}\left[\mathrm{~cm}^{-2}\left(\mathrm{~K} \mathrm{~km} \mathrm{~s}^{-1}\right)^{-1}\right]$ and a correction factor of 1.36 to account for heavy elements

$M_{\mathrm{H}_{2}}\left[M_{\odot}\right]=4.4 \pi R^{2}[\mathrm{pc}] I_{\mathrm{CO}(1-0)}\left[\mathrm{K} \mathrm{km} \mathrm{s}^{-1}\right]$,

where the source's radius $R$ corresponds to the $\mathrm{CO}(1-0)$ beamsize radius at the distance of the source $(17.5 \mathrm{Mpc}$ from Mei et al. 2007). 

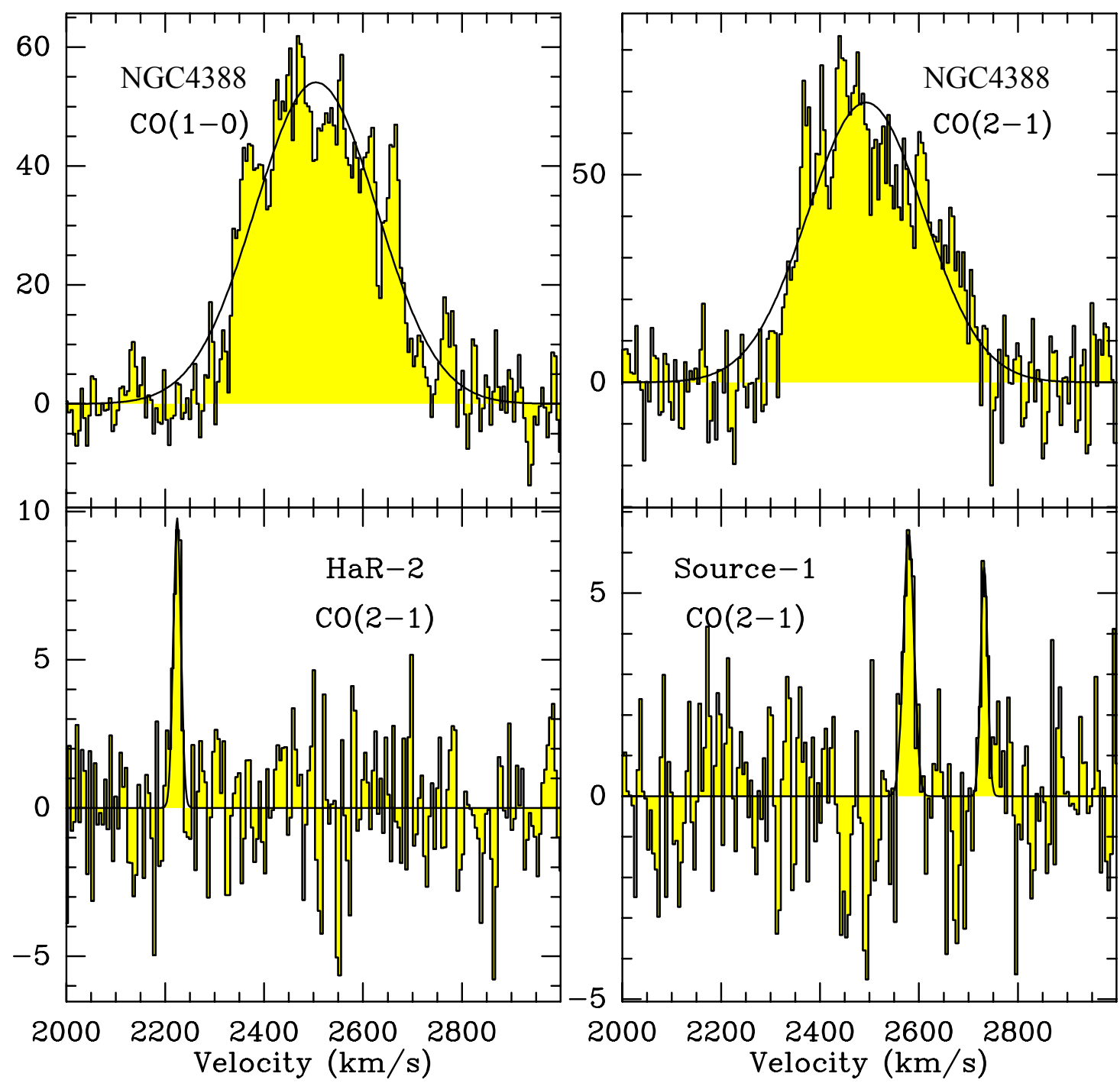

Fig. 2. Top: spectra taken at the center of NGC 4388, observed for calibration purposes. Bottom: spectra from observing run 195-13, which showed $\mathrm{CO}$ emission. Spectra information and line parameters are presented in Table 2. The temperature scale corresponds to main beam temperature in $\mathrm{mK}$.

\subsection{Star formation efficiency}

To estimate how fast is the gas being transformed into stars, we compare the SFR surface density versus the gas surface density in a $\mathrm{S}-\mathrm{K}$ relation, to understand the efficiency of this star formation process. Since these are low gas density regions, we can expect a large amount of gas in atomic phase, which is greater than in molecular phase. Therefore, we need to take both components, atomic and molecular, into account when estimating a total amount of gas to be converted into stars.

Molecular gas can be directly estimated from the $\mathrm{CO}(1-0)$ line intensity, obtained from our observations. If we take Eq. (1), and we divide it by the source area $\left(\pi R^{2}\right)$, we obtain the $\mathrm{H}_{2}$ surface density,

$$
\Sigma_{\mathrm{H}_{2}}\left[M_{\odot} \mathrm{pc}^{-2}\right]=4.4 I_{\mathrm{CO}(1-0)}\left[\mathrm{K} \mathrm{km} \mathrm{s}^{-1}\right]
$$

These values are listed in Col. 2 of Table 4 for the sources with $\mathrm{CO}(1-0)$ detections, including upper limits for the sources with no CO detection using Table 3.

For the atomic gas component, we estimated the amount of HI from the HI column density map of the NGC 4388 plume from Oosterloo \& van Gorkom (2005). The atomic gas mass is derived from the integrated amount of $N_{\mathrm{H}}$ inside the source solid angle,

$M_{\mathrm{HI}}=\mu m_{\mathrm{H}} \int N_{\mathrm{H}} \mathrm{d} A=\mu m_{\mathrm{H}} D^{2} \int N_{\mathrm{H}} \mathrm{d} \Omega$.

Aperture photometry was done in the $N_{\mathrm{H}}$ map of Oosterloo \& van Gorkom (2005) to obtain the integrated column densities for our sources. We used $22^{\prime \prime}$ apertures to be consistent with our $\mathrm{CO}(1-0)$ observations. Since these apertures are smaller than the spatial resolution of the HI map $(18 \times 95.1 \mathrm{arcsec})$, the photometry results are equivalent to the pixel value of the HI map at the position of our CO targets. These values are listed in Col. 3 of Table 4 . Then, by dividing Eq. (3) by the $\mathrm{CO}(1-0)$ beam solid angle $\Omega=\pi R^{2}$, we obtain the HI surface densities $\Sigma_{\mathrm{HI}}$ listed in Col. 4 of Table 4 .

Finally, we estimate the SFR surface density $\Sigma_{\mathrm{SFR}}$ directly from the $\mathrm{H}_{\alpha}$ emission in these regions. From Kennicutt \& Evans (2012),

$\log \operatorname{SFR}\left[M_{\odot} \mathrm{yr}^{-1}\right]=\log L_{\mathrm{H}_{\alpha}}\left[\operatorname{erg~s}^{-1}\right]-41.27$,

which, divided by the $\mathrm{CO}(1-0)$ beam solid angle $\Omega$, gives the SFR surface density $\Sigma_{\text {SFR }}$. The $\mathrm{H}_{\alpha}$ luminosities for HaR-1, 

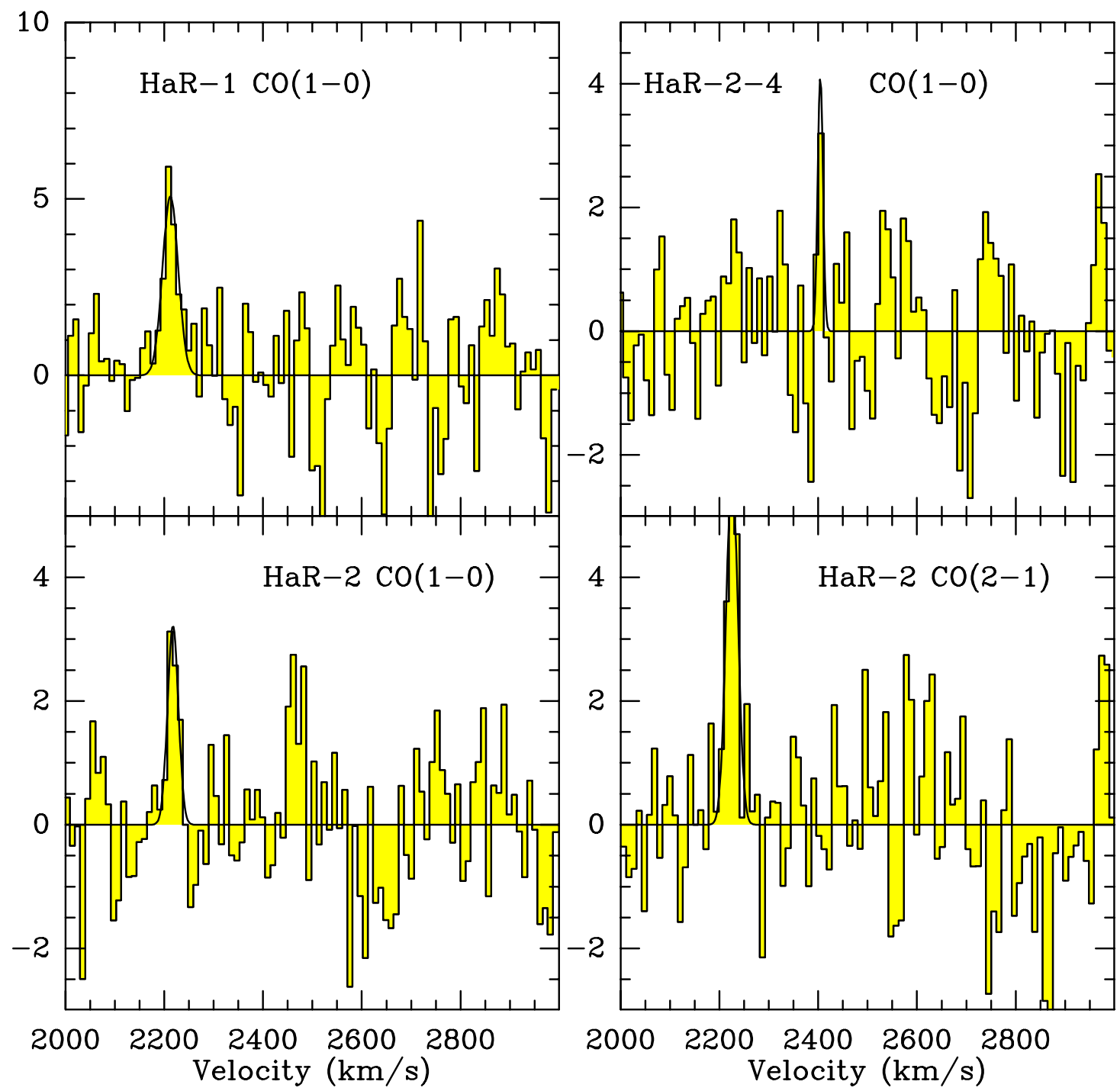

Fig. 3. Final CO spectra for HaR-2, HaR-1, and HaR-2-4 from run 075-14. In CO(2-1), only HaR-2 has data combined from both runs. All spectra have both polarizations combined. Spectra information and line parameters are presented in Table 2. The temperature scale corresponds to main beam temperature in $\mathrm{mK}$.

HaR-2, and HaR-3-4 were obtained from Yagi et al. (2013), and are listed in Table 4, along with the corresponding $\Sigma_{\mathrm{SFR}}$. For the remaining sources, we used the $\mathrm{H}_{\alpha}$ map from Kenney et al. (2008), using an aperture photometry of $\sim 1^{\prime \prime}$ in diameter, similar to the seeing of the $\mathrm{H}_{\alpha}$ observations from Yagi et al. (2013). For the sources with no visible detection in this map, an upper limit was calculated form the noise level of Kenney's map, estimated in $4.8 \times 10^{-7} \mathrm{erg} \mathrm{s}^{-1} \mathrm{~cm}^{-2} \mathrm{sr}^{-1}$.

The SFR surface densities are plotted as a function of the gas surface densities to construct a S-K relation in Fig. 4, using the values from Table 4 . We have separately plotted, the atomic and molecular gas component of $\Sigma_{\text {gas }}$, along with a plot of both components combined, in red, black, and blue markers, respectively. Arrows denote the upper limits values from Table 4. In this figure, adapted from Jáchym et al. (2014), we can compare our sources with those of Jáchym et al. in the Norma Cluster, as well as with the sample of spiral galaxies from Bigiel et al. (2008) (in colored contours), and the sample of 30 nearby galaxies from Bigiel et al. (2011), plotted as the running medians of $\Sigma_{\mathrm{SFR}}$ as a function of $\Sigma_{\mathrm{H}_{2}}$, and with a typical depletion time of $\sim 2.3 \mathrm{Gyr}$. Additionally, we included shaded ovals to represent regions from the outskirts of XUV disk galaxies. NGC 4625 and NGC 6946 data were taken from Watson et al. 2014 (priv. comm.), including IRAM-30 m CO observations and $\mathrm{H}_{\alpha}$ luminosities measured within a 6" aperture. M63 (NGC 5055) data corresponds to the bright UV region located at $1.35 \mathrm{r}_{25}$ in Dessauges-Zavadsky et al. (2014), with IRAM 30-m CO data, and a SFR measured from the FUV and $24 \mu \mathrm{m}$ emission.

"Isochrones" of constant star formation efficiencies are also shown to indicate the depletion times of $10^{8}, 10^{9}$, and $10^{10}$ years to consume all the gas, including an additional red isochrone to mark the age of the Universe as one Hubble time (i.e., $\tau_{\text {dep }}=$ 13.8 Gyr).

Contrary to the photometry done in the $\mathrm{HI}$ data, the $\mathrm{H}_{\alpha}$ data was not measured in a $22^{\prime \prime}$ diameter aperture, as the $\mathrm{CO}(1-0)$ FWHM, but in an aperture of $1^{\prime \prime}$ in diameter, similar to the seeing of those observations. Since we are averaging this $\mathrm{H}_{\alpha}$ emission in a $22^{\prime \prime}$ aperture to calculate the $\Sigma_{\mathrm{SFR}}$, we could be diluting the real surface density of the gas being converted into stars. To correct for this beam dilution, as a representation, we shifted one of the points in Fig. 4 to a fictitious $\Sigma_{\mathrm{H}_{2}}$, corresponding to a source's solid angle of $1^{\prime \prime}$ in diameter. This correction translates 
Table 2. CO detections and their line parameters.

\begin{tabular}{ccccccccccc}
\hline \hline Source & Line & Backend & $\begin{array}{c}\text { Int. time } \\
{[\mathrm{min}]}\end{array}$ & $\begin{array}{c}v_{0} \\
{\left[\mathrm{~km} \mathrm{~s}^{-1}\right]}\end{array}$ & $\begin{array}{c}F W H M \\
{\left[\mathrm{~km} \mathrm{~s}^{-1}\right]}\end{array}$ & $\begin{array}{c}\text { Resol. } \\
{\left[\mathrm{km} \mathrm{s}^{-1}\right]}\end{array}$ & $\begin{array}{c}T_{\mathrm{mb}} \\
{[\mathrm{mK}]}\end{array}$ & $\begin{array}{c}\mathrm{rms} \\
{[\mathrm{mK}]}\end{array}$ & $\begin{array}{c}I_{\mathrm{CO}} \\
{\left[\mathrm{mK} \mathrm{km} \mathrm{s}^{-1}\right]}\end{array}$ & $\begin{array}{c}M_{\mathrm{H}_{2}} \\
{\left[10^{6} M_{\odot}\right]}\end{array}$ \\
\hline HaR-2 & $\mathrm{CO}(2-1)$ & FTS & 268 & $2224 \pm 2$ & $18 \pm 3$ & 4 & 9.8 & 2.0 & $186 \pm 29$ \\
Source-1(1) & $\mathrm{CO}(2-1)$ & WILMA & 232 & $2579 \pm 2$ & $25 \pm 5$ & 5 & 6.4 & 1.7 & $173 \pm 29$ \\
Source-1(2) & $\mathrm{CO}(2-1)$ & WILMA & 232 & $2732 \pm 2$ & $16 \pm 5$ & 5 & 5.6 & 1.7 & $99 \pm 25$ & \\
\hline HaR-2 & $\mathrm{CO}(1-0)$ & WILMA & 318 & $2218 \pm 4$ & $26 \pm 7$ & 10 & 3.2 & 1.5 & $88 \pm 25$ & 1.1 \\
& $\mathrm{CO}(2-1)$ & WILMA & 586 & $2226 \pm 2$ & $24 \pm 6$ & 10 & 6.3 & 1.6 & $163 \pm 29$ & 2.4 \\
HaR-1 & $\mathrm{CO}(1-0)$ & WILMA & 229 & $2212 \pm 4$ & $35 \pm 13$ & 10 & 5.2 & 2.3 & $199 \pm 54$ & 2.3 \\
HaR-2-4 & $\mathrm{CO}(1-0)$ & WILMA & 665 & $2404 \pm 2$ & $12 \pm 3$ & 10 & 4.0 & 1.4 & $54 \pm 16$ & 0.7 \\
& & & & & & & & & \\
NGC 4388 & $\mathrm{CO}(1-0)$ & WILMA & 87 & $2505 \pm 2$ & $289 \pm 5$ & 5 & 54.0 & 4.8 & $17000 \pm 276$ & 205 \\
& $\mathrm{CO}(2-1)$ & WILMA & 82 & $2493 \pm 4$ & $275 \pm 7$ & 5 & 67 & 8.9 & $20000 \pm 490$ & \\
\hline
\end{tabular}

Notes. First three rows correspond to observing run 195-13. Both components shown in Fig. 2 for Source-1 are listed. $\mathrm{H}_{2}$ masses were calculated as $\mathrm{M}_{\mathrm{H}_{2}}\left[M_{\odot}\right]=4.4 \pi R^{2}[\mathrm{pc}] I_{\mathrm{CO}}\left[\mathrm{K} \mathrm{km} \mathrm{s}^{-1}\right]$, which uses a Galactic $\mathrm{CO}$ conversion factor of $2.0 \times 10^{20}\left[\mathrm{~cm}^{-2}\left(\mathrm{~K} \mathrm{~km} \mathrm{~s}^{-1}\right)^{-1}\right]$ and a correction factor of 1.36 to account for heavy elements. A distance to the Virgo Cluster of $17.5 \mathrm{Mpc}$ (Mei et al. 2007) was used to calculate the CO(1-0) beam radius as source radius. HaR-2 includes data taken in the first run only for $\mathrm{CO}(1-0)$, and data combined from both runs for $\mathrm{CO}(2-1)$.

Table 3. $\mathrm{CO}(1-0)$ upper limits at $3 \sigma$ for sources with no detection.

\begin{tabular}{cccccc}
\hline \hline Source & $\begin{array}{c}\text { Int. time } \\
{[\mathrm{min}]}\end{array}$ & $\begin{array}{c}\mathrm{rms} \\
\mathrm{mK}\end{array}$ & $\begin{array}{c}I_{\mathrm{CO}}{ }^{a} \\
{\left[\mathrm{~K} \mathrm{~km} \mathrm{~s}^{-1}\right]}\end{array}$ & $\begin{array}{c}M_{\mathrm{H}_{2}} \\
{\left[10^{6} M_{\odot}\right]}\end{array}$ & $\begin{array}{c}\Sigma_{\mathrm{H} 2} \\
{\left[M_{\odot} \mathrm{pc}^{-2}\right]}\end{array}$ \\
\hline Source-2 & 173 & 1.2 & $<0.11$ & $<1.21$ & $<0.48$ \\
Source-3 & 186 & 1.0 & $<0.09$ & $<0.99$ & $<0.40$ \\
Source-5 & 231 & 1.2 & $<0.11$ & $<1.21$ & $<0.48$ \\
HaR-3-4 & 229 & 1.0 & $<0.09$ & $<0.99$ & $<0.40$ \\
HaR-2-1 & 269 & 1.1 & $<0.10$ & $<1.10$ & $<0.44$ \\
HaR-2-2 & 334 & 1.1 & $<0.10$ & $<1.10$ & $<0.44$ \\
HaR-2-3 & 374 & 0.6 & $<0.05$ & $<0.55$ & $<0.22$ \\
\hline
\end{tabular}

Notes. ${ }^{(a)}$ We assumed a $\Delta v$ of $30 \mathrm{~km} \mathrm{~s}^{-1}$.

Table 4. Schmidt-Kennicutt relation values.

\begin{tabular}{ccccccc}
\hline \hline Source & $\begin{array}{c}\Sigma_{\mathrm{H}_{2}} \\
{\left[M_{\odot} \mathrm{pc}^{-2}\right]}\end{array}$ & $\begin{array}{c}\int N_{\mathrm{HI}} \mathrm{d} \Omega^{a} \\
{\left[10^{12} \mathrm{~cm}^{-2}\right]}\end{array}$ & $\begin{array}{c}\Sigma_{\mathrm{HI}} \\
{\left[M_{\odot} \mathrm{pc}^{-2}\right]}\end{array}$ & $\begin{array}{c}\log \left(L_{\mathrm{H}_{\alpha}}\right)^{b} \\
{\left[\mathrm{erg} \mathrm{s}^{-1}\right]}\end{array}$ & $\begin{array}{c}\log \left(\Sigma_{\mathrm{SFR}}\right) \\
{\left[M_{\odot} \mathrm{yr}^{-1} \mathrm{kpc}^{-2}\right]}\end{array}$ & $\begin{array}{c}\tau_{\text {dep }}\left(\mathrm{H}_{2}\right) \\
{[\mathrm{yr}]}\end{array}$ \\
\hline Source-1 & 0.93 & 0.33 & 0.40 & $<35.51$ & $<-6.19$ & $>1.4 \times 10^{12}$ \\
Source-2 & $<0.48$ & 0.41 & 0.50 & $<35.51$ & $<-6.19$ & - \\
Source-3 & $<0.40$ & 0.56 & 0.68 & $<35.51$ & $<-6.19$ & - \\
Source-5 & $<0.48$ & 0.60 & 0.69 & 37.51 & -4.20 & $<7.6 \times 10^{9}$ \\
HaR-2 & 0.39 & 2.12 & 2.5 & 37.10 & -4.61 & $1.6 \times 10^{10}$ \\
HaR-3-4 & $<0.40$ & - & - & 37.75 & -3.96 & $<3.6 \times 10^{9}$ \\
HaR-2-1 & $<0.44$ & 5.56 & 6.77 & $<35.51$ & $<-6.19$ & - \\
HaR-2-2 & $<0.44$ & 5.13 & 6.24 & $<35.51$ & $<-6.19$ & - \\
HaR-2-3 & $<0.22$ & 3.67 & 4.46 & $<35.51$ & $<-6.19$ & - \\
HaR-2-4 & 0.24 & 3.08 & 3.74 & $<35.51$ & $<-6.19$ & $>3.7 \times 10^{11}$ \\
HaR-1 & 0.88 & 2.05 & 2.49 & 35.89 & -5.82 & $5.8 \times 10^{11}$ \\
\hline
\end{tabular}

Notes. Surface densities consider a solid angle $\Omega=2.74 \mathrm{kpc}^{2}$, equivalent to the $\mathrm{CO}(1-0)$ beamsize at the distance of the source (17.5 Mpc fom Mei et al. 2007). ${ }^{(a)}$ From Oosterloo \& van Gorkom (2005). ${ }^{(b)}$ From Yagi et al. (2013) and Kenney et al. (2008).

in a +2.68 shift in log space, and is a representation of the real gas surface density at which stars would be formed, but it would always have the same $\tau_{\text {dep }}$.

For Source-5, we have made the assumption that the HI and $\mathrm{H} \alpha$ emission are spatially correlated, i.e., that they both belong to the gas plume associated with NGC 4388. We know that this is true for HI, since we used the data from Oosterloo \& van Gorkom (2005) and they probed the physical asociation of the HI gas plume to NGC 4388. This could not be the case for $\mathrm{H} \alpha$, since we used the data from Kenney et al. (2008) and their $\mathrm{H} \alpha$ map show that this source could be associated with M86 instead, when $\mathrm{H} \alpha$ is considered.
From Fig. 4, we can see that our sources have extremely low SFRs in comparison with the nearby spiral galaxies, and are only comparable with the most outer clumps in the $\mathrm{H}_{\alpha} / \mathrm{X}$-ray tail of the ISM stripped galaxy ESO137-001 in the Norma Cluster (Jáchym et al. 2014) and the XUV disk galaxies from Watson et al. (2014) and Dessauges-Zavadsky et al. (2014). We obtain depletion times that are significantly large. For example, HaR-1 and HaR-2 have $\tau_{\text {dep }}$ values of $2.2 \times 10^{12}$ and $1.2 \times 10^{11}$ years, respectively, to consume all the amount of gas present $\left(\mathrm{HI}+\mathrm{H}_{2}\right)$. These values transform into $1.6 \times 10^{12}$ and $1.0 \times 10^{11}$ years if we only consider the atomic gas component, and into $5.8 \times 10^{11}$ and $1.6 \times 10^{10}$ years if we only consider the molecular component. 


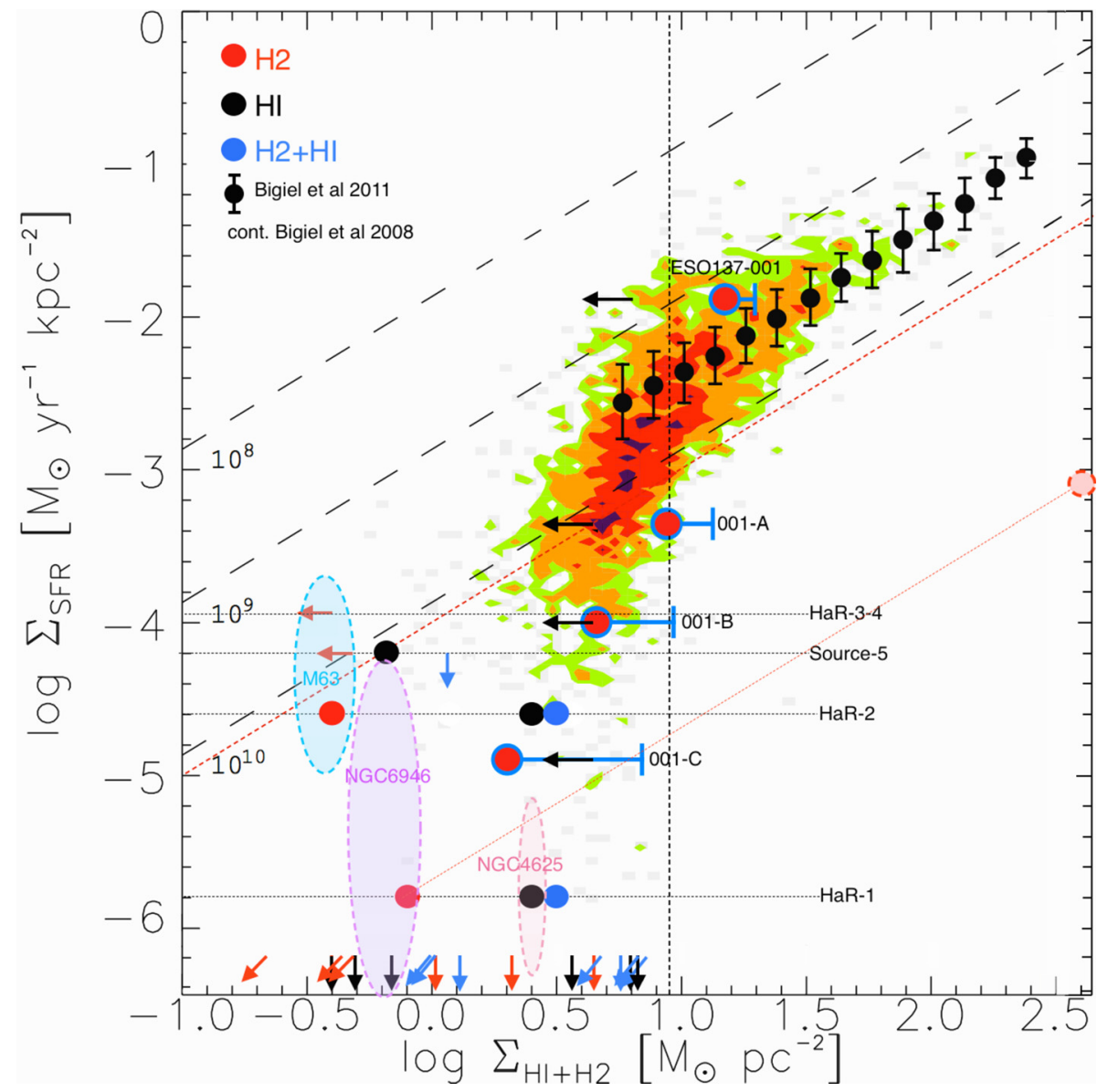

Fig. 4. S-K relation for sources in Table 4, with filled circles and arrows for values and upper limits, respectively. Red markers indicate $\mathrm{H}_{2}$ for $\Sigma_{\text {gas }}$, black markers HI, and blue ones the sum of both. Figure adapted from Jáchym et al. (2014), where their sources ESO137-001, 001-A, 001-B, and 001-C were plotted in a similar way as ours: red circles for $\mathrm{H}_{2}$ gas, black left arrows for $\mathrm{HI}$ upper limits, and blue "error bars" to account for both gas components. Colored contours account for the spiral galaxies from Bigiel et al. 2008 (green, orange, red, and purple for 1, 2, 5, and 10 sampling points per 0.05 dex, respectively). Black markers with error bars correspond to the running medians in $\Sigma_{\mathrm{SFR}}$ as a function of $\sigma_{\mathrm{H}_{2}}$ of 30 nearby galaxies from Bigiel et al. (2011). Shaded ovals represent the data from the outer parts of XUV disk galaxies: NGC 4625 and NGC 6946 from Watson et al. (2014, priv. comm.), and M63 (NGC 5055) from Dessauges-Zavadsky et al. (2014), only taking $\mathrm{H}_{2}$ into account in all of them. The dashed vertical line shows the $9 M_{\odot} \mathrm{pc}^{-2}$ threshold at which the atomic gas saturates. Dashed inclined lines represent "isochrones" of constant star formation efficiencies, indicating the depletion times $\tau_{\text {dep }}=\Sigma_{\text {gas }} / \Sigma_{\mathrm{SFR}}$ of $10^{8}, 10^{9}$, and $10^{10}$ years to consume all the gas. The dashed red isochrone marks a depletion time equal to the age of the Universe, as one Hubble time $(13.8 \mathrm{Gyr})$. A representative shift of the HaR-1 marker for $\Sigma_{\mathrm{H}_{2}}$ is drawn, to show the "effective" molecular gas density at which stars would form in this region if we consider a beam correction factor of +2.68 in log space, to convert our $22^{\prime \prime}$ beam to a $\sim 1$ " beam as in the $\mathrm{H}_{\alpha}$ data.

These values are quite large in comparison with the typical $\tau_{\text {dep }}$ of 2 Gyr for spiral galaxies, and are even larger than a Hubble time by up to 2 orders of magnitude. In Table 4, the depletion times of $\mathrm{H}_{2}$ that can be calculated are listed in Col. 7 .

The extremely low SF efficiency of our sources seems to fall off the linearity of the S-K relation for typical spiral galaxies at higher gas densities, a result previously reported in other low gas density environments, such as XUV disk galaxies (Dessauges-Zavadsky et al. 2014). Watson et al. (2014) present a different conclusion for their results in XUVs, with a typical SFR in agreement with the S-K linear regime, but they take the $24 \mu \mathrm{m}$ emission in the SFR into account, and neglect the contribution of heavy elements in the $\Sigma_{\mathrm{H}_{2}}$. We see that when we correct by these differences to make their data analytically compatible with ours (i.e. neglect the $24 \mu \mathrm{m}$ emission and correct for heavy elements), their data points in the S-K plot are comparable to ours.

\section{Summary and conclusions}

$\mathrm{CO}(1-0)$ and $\mathrm{CO}(2-1)$ observations were done with the IRAM $30-\mathrm{m}$ telescope in a total of 11 targets all along the ram- pressure stripped tail northeast NGC 4388 in the Virgo Cluster to probe the presence of molecular gas under extreme conditions. These targets were selected for having strong peaks of $\mathrm{HI}$ and $\mathrm{H} \alpha$ emission.

Four of such positions showed CO detections, and three of them concentrated in the HaR-2 region, at a distance of $\sim 70 \mathrm{kpc}$ of NGC 4388, where molecular gas in unexpected. Given the large distances of these sources to NGC 4388, it is not likely that the molecular gas was stripped from the galaxy, and must have formed in situ from the HI gas plume.

Gaussian line profiles were fitted to the spectra of the detections, finding a range of velocity dispersion between 12 and $35 \mathrm{~km} \mathrm{~s}^{-1}$. The $\mathrm{CO}(1-0)$ line profiles were used to estimate molecular gas masses and surface densities. The amount of molecular gas in these three regions (HaR-1, HaR-2 and HaR-2-4) is very low ( between 0.7 and $2.4 \times 10^{6} M_{\odot}$ ), and their $\mathrm{H}_{2}$ surface densities between 0.2 and $0.9 M_{\odot} \mathrm{pc}^{-2}$. These values are well below the $\mathrm{HI}-\mathrm{H}_{2}$ threshold, where the gas is mainly atomic and very little is know about the SFR at these low gas densities, and, hence, the importance of these detections. 
Using complementary data from Yagi et al. (2013) and Kenney et al. (2008) for $\mathrm{H} \alpha$ and from Oosterloo \& van Gorkom (2005) for HI, we computed $\Sigma_{\mathrm{SFR}}$ and $\Sigma_{\mathrm{HI}}$ to plot, in combination with $\Sigma_{\mathrm{H}_{2}}$, a S-K relation. Our sources show an extremely low SFR (up to 2 order of magnitude lower than for typical spiral galaxies). For example, HaR-1 and HaR-2 have total gas depletion times of $2.2 \times 10^{12}$ and $1.2 \times 10^{11}$ years, respectively. If we consider just the molecular gas component, these depletion times are $5.8 \times 10^{11}$ and $1.6 \times 10^{10}$ years. Furthermore, Source- 1 and HaR-2-4 have $\mathrm{H}_{2}$ depletion times even greater than $1.4 \times 10^{12}$ and $3.7 \times 10^{11}$ years respectively. These high values of depletion times exceed by far a Hubble time, thus indicating that this molecular gas will not eventually form stars, but will remain in a gaseous phase and later join the ICM.

From Fig. 4, we can see that the linearity between the SFR and the gas surface density at high gas surface densities (>9 $M_{\odot} \mathrm{pc}^{-2}$ ) for normal spiral galaxies, cannot be extrapolated to lower densities, below the $\mathrm{HI}-\mathrm{H}_{2}$ threshold, where the star formation is extremely inefficient, and the molecular gas, even though present, does not necessarily form stars.

Acknowledgements. We warmly thank the referee for constructive comments and suggestions. Also, the IRAM staff is gratefully acknowledged for their help in the data acquisition. We thank T. Oosterloo and J. Kenney for facilitating important $\mathrm{HI}$ and $\mathrm{H}_{\alpha}$ data, respectively. F.C. acknowledges the European Research Council for the Advanced Grant Program Number 267399-Momentum. We made use of the NASA/IPAC Extragalactic Database (NED), and of the HyperLeda database. C.V. acknowledges financial support from CNRS and CONICYT through agreement signed on December 11th 2007.

\section{References}

Bigiel, F., Leroy, A., Walter, F., et al. 2008, AJ, 136, 2846

Bigiel, F., Leroy, A. K., Walter, F., et al. 2011, ApJ, 730, L13

Boissier, S., Gil de Paz, A., Boselli, A., et al. 2008, ApJ, 681, 244

Boissier, S., Boselli, A., Duc, P.-A., et al. 2012, A\&A, 545, A142

Braine, J., Lisenfeld, U., Due, P.-A., \& Leon, S. 2000, Nature, 403, 867

Casasola, V., Hunt, L., Combes, F., \& Garcia-Burillo, S. 2015, A\&A, 577, A135

Cayatte, V., van Gorkom, J. H., Balkowski, C., \& Kotanyi, C. 1990, AJ, 100, 604

Chung, A., van Gorkom, J. H., Kenney, J. D. P., \& Vollmer, B. 2007, ApJ, 659, L115

Chung, A., van Gorkom, J. H., Kenney, J. D. P., Crowl, H., \& Vollmer, B. 2009, AJ, 138, 1741

Cluver, M. E., Appleton, P. N., Boulanger, F., et al. 2010, ApJ, 710, 248

Conselice, C. J., Gallagher, III, J. S., \& Wyse, R. F. G. 2001, AJ, 122, 2281

Cortese, L., Marcillac, D., Richard, J., et al. 2007, MNRAS, 376, 157

Dasyra, K. M., Combes, F., Salomé, P., \& Braine, J. 2012, A\&A, 540, A112

Davies, J. I., Bianchi, S., Cortese, L., et al. 2012, MNRAS, 419, 3505
DeMaio, T., Gonzalez, A. H., Zabludoff, A., Zaritsky, D., \& Bradač, M. 2015, MNRAS, 448, 1162

Dessauges-Zavadsky, M., Verdugo, C., Combes, F., \& Pfenniger, D. 2014, A\&A, 566, A 147

Edge, A. C., Oonk, J. B. R., Mittal, R., et al. 2010, A\&A, 518, L46

Fabian, A. C., Sanders, J. S., Taylor, G. B., et al. 2006, MNRAS, 366, 417

Feldmeier, J. J., Mihos, J. C., Morrison, H. L., Rodney, S. A., \& Harding, P. 2002, ApJ, 575, 779

Ferland, G. J., Fabian, A. C., Hatch, N. A., et al. 2009, MNRAS, 392, 1475

Gavazzi, G., Boselli, A., Mayer, L., et al. 2001, ApJ, 563, L23

Gu, L., Yagi, M., Nakazawa, K., et al. 2013, ApJ, 777, L36

Gunn, J. E., \& Gott, III, J. R. 1972, ApJ, 176, 1

Iwasawa, K., Wilson, A. S., Fabian, A. C., \& Young, A. J. 2003, MNRAS, 345, 369

Jáchym, P., Palouš, J., Köppen, J., \& Combes, F. 2007, A\&A, 472, 5

Jáchym, P., Combes, F., Cortese, L., Sun, M., \& Kenney, J. D. P. 2014, ApJ, 792, 11

Kenney, J. D. P., \& Young, J. S. 1989, ApJ, 344, 171

Kenney, J. D. P., van Gorkom, J. H., \& Vollmer, B. 2004, AJ, 127, 3361

Kenney, J. D. P., Tal, T., Crowl, H. H., Feldmeier, J., \& Jacoby, G. H. 2008, ApJ, 687, L69

Kennicutt, R. C., \& Evans, N. J. 2012, ARA\&A, 50, 531

Leroy, A. K., Walter, F., Sandstrom, K., et al. 2013, AJ, 146, 19

Lim, J., Ohyama, Y., Chi-Hung, Y., Dinh-V-Trung, \& Shiang-Yu, W. 2012, ApJ, 744, 112

Machacek, M. E., Jones, C., \& Forman, W. R. 2004, ApJ, 610, 183

Machacek, M., Dosaj, A., Forman, W., et al. 2005, ApJ, 621, 663

Mahdavi, A., Geller, M. J., Fabricant, D. G., et al. 1996, AJ, 111, 64

Mei, S., Blakeslee, J. P., Côté, P., et al. 2007, ApJ, 655, 144

Merritt, D. 1984, ApJ, 276, 26

Mihos, J. C., Harding, P., Feldmeier, J., \& Morrison, H. 2005, ApJ, 631, L41

Nulsen, P. E. J. 1982, MNRAS, 198, 1007

Oosterloo, T., \& van Gorkom, J. 2005, A\&A, 437, L19

O'Sullivan, E., Giacintucci, S., Vrtilek, J. M., Raychaudhury, S., \& David, L. P. 2009, ApJ, 701, 1560

Quilis, V., Moore, B., \& Bower, R. 2000, Science, 288, 1617

Roediger, E., \& Hensler, G. 2005, A\&A, 433, 875

Salomé, P., Combes, F., Edge, A. C., et al. 2006, A\&A, 454, 437

Salomé, P., Combes, F., Revaz, Y., et al. 2011, A\&A, 531, A85

Scott, T. C., Cortese, L., Brinks, E., et al. 2012, MNRAS, 419, L19

Serra, P., Koribalski, B., Duc, P.-A., et al. 2013, MNRAS, 428, 370

Sun, M., Donahue, M., \& Voit, G. M. 2007, ApJ, 671, 190

Sun, M., Donahue, M., Roediger, E., et al. 2010, ApJ, 708, 946

Tamura, T., Maeda, Y., Mitsuda, K., et al. 2009, ApJ, 705, L62

Tonnesen, S., Bryan, G. L., \& van Gorkom, J. H. 2007, ApJ, 671, 1434

Vollmer, B., Cayatte, V., Balkowski, C., \& Duschl, W. J. 2001, ApJ, 561, 708

Vollmer, B., Braine, J., Combes, F., \& Sofue, Y. 2005, A\&A, 441, 473

Vollmer, B., Beckert, T., \& Davies, R. I. 2008, A\&A, 491, 441

Watson, L. C., Martini, P., Lisenfeld, U., et al. 2014, in AAS Meet. Abst., 223, 454.22

Yagi, M., Komiyama, Y., Yoshida, M., et al. 2007, ApJ, 660, 1209

Yagi, M., Gu, L., Fujita, Y., et al. 2013, ApJ, 778, 91

Yoshida, M., Yagi, M., Okamura, S., et al. 2002, ApJ, 567, 118

Zhang, B., Sun, M., Ji, L., et al. 2013, ApJ, 777, 122 